Voix et Images

volxetimages

\title{
Naïm Kattan romancier : La Promesse du temps retrouvé
}

\section{Sylvain Simard}

Volume 11, numéro 1, automne 1985

\section{Naïm Kattan}

URI : https://id.erudit.org/iderudit/200535ar

DOI : https://doi.org/10.7202/200535ar

Aller au sommaire du numéro

Éditeur(s)

Université du Québec à Montréal

ISSN

0318-9201 (imprimé)

1705-933X (numérique)

Découvrir la revue

Citer cet article

Simard, S. (1985). Naïm Kattan romancier : La Promesse du temps retrouvé.

Voix et Images, 11(1), 33-44. https://doi.org/10.7202/200535ar d'utilisation que vous pouvez consulter en ligne.

https://apropos.erudit.org/fr/usagers/politique-dutilisation/ 


\title{
Naïm Kattan romancier : La Promesse du temps retrouvé
}

\author{
par Sylvain Simard, Université d'Ottawa
}

Tout en l'assurant du respect et de l'estime du milieu littéraire et de la fidélité d'un certain public, l'œuvre de Naïm Kattan reste encore marginale, en tout cas mal connue dans notre littérature. À part quelques analyses ponctuelles, on semble ne pas trop savoir par quel bout prendre ces livres, romans, essais, nouvelles, qui échappent en apparence du moins, à toutes les catégories bien connues de notre littérature autochtone. Et même s'il a trouvé à se faire une petite place littéraire à Paris où plusieurs critiques, parmi les meilleurs, ont souligné son talent, les quelques articles qui lui sont consacrés ne vont guère au-delà de la surprise devant l'originalité et l'exotisme de l'œuvre.

En effet, on ne peut pas ne pas le rappeler, Naïm Kattan ne vient ni de Kamouraska ni du Plateau Mont-Royal. Arrivé au Canada, il y a 30 ans, ce judéo-arabe irakien est né à Bagdad en 1928. Après des études de lettres à Paris, il descend, un froid matin d'hiver 1954, du train Halifax-Montréal, décidé à vivre en ce pays. Depuis, il écrit; d'abord dans les journaux et revues littéraires et depuis une quinzaine d'années, des livres, essais, critiques littéraires, romans, nouvelles et même poésie.

L'embarras de la critique québécoise est compréhensible. Naïm Kattan a décidé d'écrire en français à Montréal: il a choisi de s'intégrer à la culture franco-québécoise tout en maintenant totalement vivante l'originalité de ses origines et la spécificité d'une vision très personnelle. Imprégné de son expérience de déraciné, il a voulu, comme mythe et comme espoir personnel, analyser la promesse et sa réalisation en terre d'Amérique. En ce sens, il n'est pas exagéré de dire qu'il n'est pas une page de Kattan essayiste ou romancier qui ne pose le problème de l'identité sous l'une ou l'autre de ses facettes. Et comme telle, puisant à des sources millénaires, cette ouvre rejoint l'un des axes les plus fertiles de la création littéraire au Québec, celui qui d'Hébert à Miron, de Godbout à Tremblay en passant par Ducharme, Pilon, Ouellette, Vadeboncoeur et plusieurs autres, ne cesse d'interroger le sens même de notre existence collective. Puisant aux grands archétypes mythiques de la Bible, soucieux de mettre sa quête à la fois candide et désespérée au diapason du chant coranique et de la mystique talmudique, Naïm Kattan essaie de formuler en termes modernes, américains et montréalais, la promesse qui autrefois fut faite à un peuple qui vécut vingt-cinq siècles à Babylone avant d'en être chassé. Cherchant à comprendre les causes et l'étendue de l'échec de l'Amérique à réaliser la promesse biblique qui lui a donné naissance, Kattan, à mesure que son œuvre progresse, semble l'investir de cette patience qui n'appartient qu'aux visionnaires et aux prophètes, celle qui dans l'absurde et le désespérant cherche à lire les signes de l'espoir. 
Aux visions claires, aux systèmes explicateurs cohérents du premier essai et à l'unité problématique mais réelle du narrateur d'Adieu Babylone, vont succéder des approches plus éclatées et un narrateur apparemment désarticulé. Comme si Naïm Kattan, à mesure qu'il avance dans son interrogation du monde, y découvrait plus de doutes que de certitudes. Sommé de s'identifier, il fait l'inventaire des mondes et des cultures auxquels il se rattache, non pour trouver une univocité du je qui ne pourrait être qu'artificielle mais pour prendre en compte sa complexité et en accepter les contradictions. La structure mềme des romans reflète très bien cette démarche tout comme leur contenu thématique renvoie aux sources mêmes de l'écriture katannienne.

Né à Bagdad, j’ai fait ma vie à Montréal. À moins de retrancher une dimension de mon être, de gommer un passé, il importait de chercher le fil conducteur qui donne au mouvement une cohérence, une continuité malgré les ruptures, les points d'arrêt et les retours.

(La Mémoire et la promesse, p. 5)

Adieu Babylone, paru en 1975, est le début d'une suite romanesque qui s'est poursuivie avec les Fruits arrachés en 1977 et la Fiancée promise en 1983. Il n'est pas impens̄able que l'auteur ajoute d'autres titres à cette trilogie: nulle part l'auteur ne mentionne qu'ils fassent partie d'une suite et le contenu narratif laisse le héros-narrateur à la fin du dernier roman en début de l'âge adulte. L'unité romanesque n'est ni géographique ni historique, chacun des titres correspondent à des lieux fort différents; le premier, c'est l'univers oriental de Bagdad, le second est européen et circonscrit surtout au Paris de la rive gauche alors que Montréal sert de matière et de décor au troisième. Les séquences chronologiques marquent davantage l'unité de la démarche puisqu'elles correspondent à des époques successives de la vie d'un même personnage-narrateur. L'Orient arabe est le lieu de l'adolescence, Paris celui des études universitaires et du passage à "l'âge d'homme" alors qu'en Amérique le jeune adulte cherche à se fixer et s'enraciner. À cette unité de point de vue narratif correspondent cependant de profondes différences structurelles. Même en feuilletant rapidement les trois ouvrages, celles-ci sautent aux yeux.

Alors qu'Adieu Babylone est fait de longs paragraphes discursifs, le second est essentiellement constitué de dialogues, tout comme le troisième, même si la part faite aux parties narratives y est dans ce cas plus grande. Les divisions internes sont par ailleurs très floues. Aux dix-huit chapitres non numérotés du premier ${ }^{1}$, dont un de soixante et dix pages et un autre de trois pages, correspond dans les Fruits arrachés, rythmé toutes les deux ou trois pages par des $*^{*} *$, un texte très rapide fait de courtes séquences. Dans la Fiancée promise ${ }^{2}$, les ruptures, discrètement soulignées, sont aussi ramenées à des changements de scènes propres au théâtre; ruptures géographiques et temporelles, apparitions-disparitions de personnages. Ces différences et ressemblances structurelles ne font que mettre en évidence les questions entourant la nature même et l'unité de ces ouvrages. 
Tous trois sous-titrés roman, on ne retrouve nulle part dans le texte, directement ou indirectement, la trace d'un pacte autobiographique ni la moindre indication sur une possible identité auteur-narrateur. Et pourtant... Pour se limiter uniquement à cette vision d'une fiction romanesque, le lecteur, singulièrement le lecteur québécois, devrait faire abstraction de toute information externe, ignorer ou oublier ce qu'il sait de Naïm Kattan. Il ne peut en tout cas l'ignorer s'il a lu l'un ou l'autre de ses essais qui puisent tous à ces réalités vécues. Et s'il en ignore tout, les textes de présentation, préfaces ou pages de garde, se chargeront de lui en apprendre l'essentiel. Kattan, dans le Désir et le pouvoir mentionne qu'il est l'auteur de récits autobiographiques et Michel Tournier, dans sa préface à l'édition française d'Adieu Babylone, mélange allègrement et sans complexe l'auteur et le narrateur et qualifie l'ouvrage de livre de souvenirs, ce qu'aucune note de l'auteur ne vient démentir: tout n'est pas parfaitement clair $^{3}$ et nous sommes manifestement ici dans une zone floue de la frontière roman-autobiographie. Il faut prendre acte du fait que l'auteur a voulu garder entière sa liberté et la possibilité de combiner à sa guise le réel et l'imaginaire, la fiction et le souvenir. Et si on s'interroge sur la vérité du texte et la sincérité des confidences, on doit admettre que le refus d'assumer totalement la réalité historique lui donne peut-être plus de liberté pour aller à l'essentiel; le masque est sans doute transparent mais il autorise le romancier à certaines audaces que l'autobiographe aurait peut-être dû camoufler. Cela est d'autant plus vrai à propos des différentes étapes de la vie sentimentale et érotique du narrateur. Il y a toutes sortes de dégradés dans les notions intermédiaires entre le roman autobiographique et l'autobiographie romancée et René, Adolphe, les Confessions ou la Recherche, ne sont en somme que des formes différentes d'apparition de ce moi si peu haïssable depuis deux siècles. Mais cette volonté de se maintenir dans la zone d'ombre de la responsabilité du texte, en plus de mener comme chez Colette à d'inévitables ambiguïtés, n'enlève-t-elle pas ce que Michel Leiris appelle la corne acérée du taureau, c'est-à-dire la plus-value littéraire et humaine du risque vital et du danger encouru? Mais, puisqu'ils apparaissent au premier abord structurellement différents, c'est le contenu de chacun des ouvrages qu'il faut interroger.

Adieu Babylone doit sans doute la fascination qu'il exerce au triple fil conducteur qui le traverse: le roman d'éducation qui raconte les ardentes découvertes littéraires, culturelles et amoureuses d'un narrateur adolescent; l'exotisme, non pas de façade mais intrinsèque à l'originalité de cet univers oriental dont la sociologie complexe fait revivre le monde aujourd'hui disparu de la communauté juive de Bagdad; historique enfin, car il veut faire l'histoire et participer activement à la création d'un état irakien au moment où le nationalisme arabe et la naissance d'Israill exacerbent un sentiment anti-juif qui ne peut aboutir qu'au départ. Adieu Babylone est aussi le lieu par excellence de la contradiction fondamentale, l'aspiration à l'enracinement dans un lieu, une nation, une langue et l'appel quasi irrésistible de l'Occident, de sa civilisation, de sa culture. Aucune autre œuvre, contemporaine, n'associe avec autant de force et de vérité ces quêtes contradictoires et leurs manifestations linguistiques; entre les dialectes arabes juif et 
musulman, la langue du Coran et celle de Gide et Aragon se joue une partie serrée puisque chacune exprime des choix culturels et politiques mutuellement exclusifs.

Le récit est simple; la vie du narrateur, de 14 à 19 ans, pendant la seconde guerre mondiale, à Bagdad. Le point de vue rétrospectif permet, à travers les événements significatifs ramenés au présent de la mémoire, de comprendre et de donner un sens à l'existence, à ce qui viendra après. Mais cette vision a posteriori n'intervient jamais directement dans le discours si ce n'est pour fournir une distanciation critique, mélange d'amour-passion et d'ironie, sur les faits et les personnages de ce passé. Passé qui revit par petites touches, sédimentation des années d'enfance, de la vie d'une famille, de la vie d'un peuple qui depuis vingt-cinq siècles cherche à maintenir son originalité et son appartenance à la terre. Babylone n'est pas seulement le champ de ruines qui rappelle la gloire passée de Nabuchodonosor II, non plus que le souvenir d'un lieu d'excursion, c'est celui où cinq siècles avant notre ère les Juifs de la tribu de Juda, après la destruction du temple, ont été amenés en esclavage; moment de l'existence d'un peuple qui a non seulement donné certaines des pages les plus belles de la Bible mais lieu de rédaction du Talmud qui a fondé une partie de la mystique juive. C'est pourquoi quitter Bagdad, c'est tourner l'une des plus longues pages de l'histoire humaine. Dire adieu à Babylone, c'est aussi dire adieu à une expérience de cohabitation avec les tribus venues du fond des âges et de tous les horizons de ce MoyenOrient; assyriens, arméniens, kurdes, bédouins, musulmans, sunnites et chiites, chrétiens, etc.

D'une structure chronologique classique, s'autorisant tout au plus quelques retours au monde de la petite enfance, le roman s'ouvre sur une scène chargée de résumer l'essentiel: au café Yassine le narrateur et ses camarades sont en pleine discussion à partir de leurs lectures du jour. Aux modèles américains de Nazar c'est la richesse de la tradition littéraire arabe qu'oppose Zaki. Mais le scandale vient de Nessim, l'ami intime, l'alter ego, à la fois confident et concurrent. Car ce soir devant ce jeune aéropage à majorité arabe musulmane, il a décidé d'utiliser le dialecte juif: intransigeant, idéaliste, extrémiste, débraillé sans arriver à dissimuler son élégance naturelle, Nessim fascine son ami plus modéré. Face à l'unanimisme mythique du narrateur (nous n'étions ni Juifs ni Musulmans mais Irakiens). Nessim fait éclater, en faisant respecter sa réalité juive, l'aspect artificiel de cet accord de façade. Comme tous les Juifs de Bagdad, il ne peut oublier la nuit du Farroud, où, laissée à elle-même la ville a vu se déchaîner ses vieux démons dans un horrible pogrome. Hors de la protection de l'État ou de l'occupant britannique, les instincts antiques de la tribu remontent toujours à la surface. Le nationalisme arabe qu'épouse sans réticence le narrateur ne peut totalement faire taire le souvenir de Farroud; désormais l'hypothèse du départ est ouverte. Kattan sait presque toujours éviter les pièges de l'exotisme en se montrant lui-même fasciné par l'exotisme du monde qui l'entoure; en faisant sien le point de vue externe, il ne cherche pas à créer un décor séduisant mais à nous entraîner à épouser son propre sentiment d'attachement fait d'amour et de distance devant ce monde oriental. Membre d'une de ses composantes 
minoritaires, il nous le décrit de ses yeux d'enfant et d'adolescent, séduit et fasciné par l'univers musulman qu'il admire et qu'il craint. Nous sommes dans l'univers de l'hostilité et du compromis car vivant à la lisière du monde musulman nous en ressentions l'étrangeté qui se transmuait souvent en exotisme (p. 42).

Cet exotisme, il nous est donné entre autres par les déplacements à travers Bagdad. Vision de la Mosquée dorée de Kadhimaine, lieu sacré des chiites, les couleurs et les parfums du marché couvert, les roses de l'Ambassade d'Angleterre, la fête de la circoncision d'enfants musulmans. Spectacle aussi de la Sbaya, cette passion chiite, remarquable récréation du souvenir, fascination mêlée de terreur de l'enfant devant la représentation de la mort lente de Hussein et Hassan, martyrs de la foi. Toujours une grande sympathie pour des moeurs qui dans leur étrangeté peuvent justifier leur propre différence. Les frontières ne sont pas qu'ethniques et linguistiques; s'y ajoute l'opposition des quartiers riches et pauvres à l'intérieur de chacune des communautés. Kattan se livre à un travail précis et subtil de description de la géographie sociologique de la ville du désert. Cet arrière-plan nous est livré sans lourdeur didactique, parfaitement intégré à la liberté du récit et des narrations et permet seul de comprendre le rôle essentiel des Juifs dans la création du nouvel état. Mais là encore c'est la précision de l'observation socio-linguistique qui fournit, parce qu'elle est vécue par le narrateur dans sa chair et n'a pas cessé d'être une donnée fondamentale de la vie de Kattan, les clefs essentielles à la compréhension du drame.

Adieu Babylone c'est aussi l'apprentissage sexuel et la découverte de l'amour dans un univers oriental où ces deux réalités doivent être vécues séparément. À l'irréalité de la femme, mère, sœur, future épouse, cachée aux regards de l'homme, répond le monde réel, celui du sexe épanoui, gourmand hors du sentiment du péché. Les prostituées dispensatrices de cette réalité renvoient à l'homme offensé l'image d'une tendresse humiliée, d'une sensibilité mutilée.

Mais surtout une vocation d'écrivain naît, s'affirme, s'épanouit. Fou de la langue et de la littérature arabe dont il veut explorer toutes les subtilités, il découvre avec naïveté et candeur les premiers plaisirs de la gloire d'être lu. Les premiers émois amoureux ne s'appuient-ils pas sur les premiers succès littéraires? Mais c'est aussi la découverte de l'Occident et des auteurs français. Au zénith de sa passion pour la culture arabe il se prépare à partir vers un ailleurs où la promesse pourra s'accomplir. Non pas Israël, mais Londres, Milan ou Paris; il ne s'agit pas de couper avec la littérature irakienne mais de la faire connaître: Au lieu d'être affadie par la perspective du départ, ma passion pour notre littérature s'en était au contraire avivée. (p. 193). Dans sa fièvre du départ, il ne veut rien sacrifier. De ces apparentes contradictions, il ne faut pas s'étonner; nous sommes dans l'univers du rêve, celui du café et des amis, univers de la pensée pure et de la littérature. Quel choc et quelle gêne pour eux d'aller chez Saïd le jour de son anniversaire. Ils y sont confrontés avec la réalité de leur propre médiocrité que chaque jour ils tentent d'abstraire et de transcender. Monde réel, fait d'odeurs, de saletés, de couleurs, univers du rituel, de l'original mais aussi de l'archaïsme et du 
préjugé, contact quotidien de cultures différentes dans une civilisation commune. Lourde présence des monstres anciens prêts à tout moment à traduire en violence la sourde haine qui les alimente. Puis tout cela ne serait que thèse et abstraction si Kattan n'avait si bien réussi à nous communiquer aussi sensuellement, par petites touches, les principales composantes d'un Orient qui séduit et repousse. Les frontières linguistiques, reflets si précis des frontières ethniques religieuses et sociales, ne se découpent pas mais se superposent. Elles ne font pas que mener le narrateur à l'âge d'homme, elles en tissent le substrat culturel et psychologique, elles définissent déjà les bases de l'écriture. Par ce retour aux rituels d'un univers disparu, Naïm comme Marcel, crée l'écrivain Kattan. L'Occident européen puis américain ajouteront mais ne déferont rien. Cette recréation du monde du départ, cette mise à jour des enjeux, ce voyage du souvenir créateur aux origines de l'homme d'aujourd'hui ne sont pas le point culminant d'une sagesse finalement acquise, d'une compréhension d'un mythique fil qui expliquerait et donnerait un sens à ce qui fut depuis la vie de l'écrivain. Aucune réponse univoque à la question des origines puisqu'elles furent le lieu du multiple et de l'ambigu: il n'est pas que juif, arabe francisé, occidentalisé. Aucune catégorie réductrice n'arrivera à dissimuler la complexité créatrice des origines et de l'expérience. Il ne faut donc pas le chercher ailleurs que dans son œuvre.

Roman trouble et troublant, les Fruits arrachés reflète bien dans sa facture le problème qui en est le centre, l'identité perdue. Contrairement à Adieu Babylone, vision rétrospective parfaitement élaborée, syntèse envoûtante du milieu des origines, la vie du jeune juif de Bagdad, cette suite à l'entreprise autobiographique ne fait que poser les problèmes sans, non seulement les résoudre, mais sans même chercher à les mettre en perspective. Nous sommes ici constamment au niveau de l'existentiel et les problèmes onthologiques loin d'être théorisés, n'existent qu'à travers les rencontres amoureuses, les amitiés, la découverte de Paris et de l'Europe. Roman sur l'identité, il pose le problème de l'identité du roman. Fait de rencontres et donc de dialogues de personnages sans épaisseur psychologique, il n'est traversé par rien d'autre qu'une quête qui n'est jamais définie. En ce sens, malgré l'envahissement d'une thématique dominante, en dépit du classicisme de son évolution chronologique et spatiale, les Fruits arrachés sont à la fois les héritiers de Camus et de Robe-Grillet. La présence lancinante de l'Orient et l'absence de signification préétablie pour le premier, une forme qui semble échapper à la domination du couple auteur-narrateur pour le second, un refus, en somme de se donner le beau rôle.

Il s'agit bien du roman de l'identité perdue: à travers les rencontres, les amitiés, les amours, les découvertes esthétiques et intellectuelles, c'est toujours pour Meïr, le jeune Juif venu de Bagdad, comme aux yeux de ses interlocuteurs, le véritable problème. La recherche de l'amour à travers des êtres très différents et la tentative de donner une définition de lui-même se fondent en un tout et cette naissance à la vie ne peut venir que de la mort. 


\begin{abstract}
Émigrer, c'est s'arracher du réel, au monde extérieur comme donnée immédiate sans pour cela faire de la nature un décor; c'est plonger lucidement dans un nouvel hiver, un autre été, oublier le goût de tous les fruits pour les découvrir à nouveau, c'est mourir pour renaître, enterrer dans la mémoire une autre vie pour qu'une autre puisse éclore.
\end{abstract}

(Le Réel et le théâtral, p. 177)

$\mathrm{Au}$ début du roman, Meïr apprend la naissance de l'État d'Israël. Aucune joie à cette annonce puisqu'elle signifie à court terme le départ forcé des Juifs d'Irak. La décision des Nations Unis vient d'en faire un apatride; sa famille sera persécutée et l'illusion qu'il a nourrie sans trop y croire de revenir vivre à Bagdad, s'effondre. Les menaces qui pèsent sur son séjour à Paris se précisent; la peur de l'échec aux examens et du non renouvellement de sa bourse, le sort de sa famille, la crainte d'être mal jugé par ses compatriotes irakiens, le besoin d'être accepté comme il est sans avoir à devenir ce que l'on attend de lui, ne pas être un Juif ou un Arabe, mais rester juif et arabe. Jusqu'à la fin, il refusera de choisir, de se couper d'une partie de ce qu'il a été et de ce qu'il veut être. Il est le correspondant parisien d'un quotidien de Bagdad même s'il doit signer ses articles d'un pseudonyme français: Irakien, il l'est et voudrait le rester. Il veut préserver sa relation avec ses compatriotes étudiants comme lui mais le fossé s’élargit de plus en plus et les détours se font de plus en plus longs pour éviter de les rencontrer à la Source, leur café habituel.

Il est oriental et ne veut rien céder de cet aspect essentiel de son être; d'où son admiration pour Camus, son plaisir irrationnel à manger dans des restaurants arabes ou à amener sa fiancée admirer le spectacle d'une danseuse du ventre. Mais il n'est pas abstraitement oriental et arabe, il est d'abord irakien, il fait partie de cette jeunesse intellectuelle qui travaille à former une culture nationale. Et c'est d'abord de la perte de cette identité dont il est menacé à la fois par la révolution politique à Bagdad et surtout par son intégration voulue et rapide à l'Occident. Paris c'est tout à la fois la ville désirée et aimée, celle qu'on explore dans ses moindres secrets; mais c'est aussi la ville déserte, le décor inanimé et indifférent qui lui apparaît hostile: il n'y a que dans la folie d'une soirée chez des amis brésiliens qu'il se retrouve comme à Bagdad. Mais son identité est menacée plus concrètement. Depuis qu'en Irak les Juifs sont accusés d'être sionnistes ou communistes, ses compatriotes de Paris deviennent de plus en plus agressifs à son égard; il le ressent d'autant plus amèrement qu'il a plus fait pour son pays qu'aucun d'entre eux. À l'annonce de l'emprisonnement de son frère, cette amertume se transforme en désespoir. Meir se raccroche quelques instants aux propos de ce haut-fonctionnaire à Genève l'assurant qu'il est encore Irakien mais son ami Nessim, désormais apatride, lui rappelle que bientôt il n'aura plus de passeport. $\grave{A}$ partir de là tout s'emballe; la panique s'empare du jeune étudiant: il voit déjà les policiens venant le chercher à son hôtel. Aussi, des mois avant l'échéance, demande-t-il le renouvellement de son passeport à des employés de consulat de plus en plus hostiles. Mais il ne renie rien de ce qu'il est; il s'adresse en arabe à ses compatriotes malgré tout ce qu'il nous ont fait. 
Les écrivains français ignorent tout de la civilisation arabe si ce n'est, comme Albert Béguin, pour traiter de l'influence mauresque en Espagne. Mais au delà de cette ignorance, c'est le refus d'être médiatisé, d'être défini à travers des catégories externes. D'ailleurs si on connaissait bien sa réalité arabe, il insisterait sur la réalité juive et les camps, car ces visions ne le rejoignent jamais dans ce qu'il est réellement, c'est-à-dire dans sa complexité indéfinie.

Ce qu'il est, cette identité profonde est davantage reconnaissable dans sa quête que dans son affirmation. Cette quête, c'est d'abord dans la rencontre amoureuse qu'elle s'incarne. Trois visages; Halina la Polonaise qui se raccroche à Paris et à Meïr avec d'autant plus de passion que le retour à Varsovie est inéluctable; Maxie la Hollandaise, la beauté nordique, celle de la différence, telle que je l'imaginais depuis ma naissance et Anne, la Française, image à la fois de la fragilité féminine mais forte de tout ce que représente sa nationalité et sa famille bourgeoise. Différemment, avec une apparente duplicité, il manifeste tous les signes de la passion pour chacune de celle à qui il déclare son amour: je déborde, (...). Je ne trouve pas d'autres mots à te dire, répond-t-il à Maxie qui lui reproche de lui dire trop souvent qu'il l'aime.

À l'ébullition normale de ses vingt ans, s'ajoute une crainte absolue de la solitude, la volonté de s'adapter à l'Occident, de se hisser aux standards français, de ne pas être réduit à la perpétuelle drague de ses amis irakiens. Avec Halina, il partage le sentiment d'avoir échappé au pire, d'être à Paris dans un hâvre de bonheur, oubliant l'inéluctable retour à Varsovie et Bagdad où tout n'est qu'inquiétude et désespoir. Si Maxie ne peut être que la blonde réponse au fantasme oriental, Anne marque l'ambiguïté du désir et du refus d'intégration à la société française. Il se sent d'ailleurs tendre, fautif; Je suis renfrogné. Vais-je dire qu'il y a Maxie et Halina? Cette vision transitoire des passions, toute l'œuvre de Naïm Kattan en est littéralement remplie.

Si les passions semblent éphémères, malgré leur violence, c'est qu'elles ne sont pas fixées dans un système intermédiaire, qui les consolide et qui leur fait perdre leur caractère de spontanéité tout en les alimentant continuellement. Elles se succèdent comme les fidélités, et elles se renouvellent. Si elles manquent de constance, elles ne perdent pas leur réalité. On dissimule mais chaque visage garde caractère et retrouve vie à la première provocation. Il revêt des états successifs.

(Le Réel et le théâtral, p. 187)

Ces états de sincérité successifs ne peuvent se détacher de la transition entre un Orient de la dualité amoureuse et un Occident des rapports égalitaires entre l'homme et la femme. Épouser Anne c'est tenter de s'intégrer à cette France où les choses sont trop riches, c'est-à-dire en place depuis trop longtemps pour s'y faire reconnaître dans son essence, hors de l'exotisme de la différence.

Meïr vit toutes ses contradictions, ses craintes, ses espoirs de façon immédiate et spontanée. Aucune analyse rétrospective qui viendrait ici clarifier le tableau, sortir le personnage et le lecteur du sentiment très net de 
s'enfoncer dans le sable, d'avancer dans le temps et de circuler dans le nouvel espace occidental sans plan, sans certitude. Ce n'est pas là que la promesse peut s'accomplir puisqu'elle y est réalisée depuis trop longtemps. Il faut se tourner vers un nouveau désert.

Sur la page de garde de la Fiancée promise l'auteur(?) a écrit: C'est surtout l'histoire, drôle à force de tristesse, d'un homme qui, en raison de sa faiblesse autant qu'en dépit d'elle, traverse imperturbablement les frontières. Il serait héroïque s'il n'était inconscient, il serait infiniment triste s'il n'était pas doué de tant de candeur et porteur de tant d'énergie dans l'attente.

Ainsi le personnage narrateur des Fruits arrachés, anti-héros subissant plus les événements qu'il ne les détermine, serait devenu un simple pantin désarticulé et pathétique, totalement bousculé par le réel sans aucune prise sur le temps. Nous sommes loin du héros balzacien d'Adieu Babylone qui tel Lucien de Rubempré se préparait à conquérir la gloire littéraire. L'intermède parisien s'il a été riche en apprentissages, de la femme à la langue française, a aussi été le lieu d'une désarticulation de l'identité. Meîr ne pourra plus avoir d'existence que problématique, fidèle à ses origines orientales et judéo-arabe mises en perpétuelle opposition dialectique avec l'Occident européen puis américain. Cet homme en devenir sort façonné, transformé mais surtout meurtri de l'expérience française: le rêve, confronté à la réalité, s'est dissipé! La force, la permanence, la richesse de cette culture et de cette civilisation l'ont ébloui et séduit mais il a compris qu'il n'avait pas sa place, inséré beaucoup trop tard dans un univers qui n'est plus fait de promesse mais d'acquis.

Nous l'avons quitté au Havre sur un bateau faisant route vers New-York en 1949 et même si nous le retrouvons dans le train Halifax-Montréal en $1954 \ldots$, le personnage est toujours le même: il ne s'est rien passé depuis les Fruits arrachés, Ce qui ne veut pas dire que la définition qu'en donne Kattan soit juste; rien de moins imperturbable que sa façon de traverser les frontières; il n'est certes pas dénué d'une certaine candeur et d'une énergie peu commune mais il n'a rien d'un inconscient: ce n'est pas parce que l'auteur lui refuse la faculté d'auto-analyse qu'il devient, tel un personnage de Beckett, attente indéterminée. En ce sens Meïr a peut-être échappé à Naïm, ce qui ne serait pas un précédent...

Il ne se passe rien ou presque dans ce roman. Arrivé à Montréal au cœur de l'hiver, nous quittons le narrateur à la fin de l'été. La recherche d'un travail, les premières rencontres et les premières amours, rien que de très banal si à nouveau chaque événement de sa vie, chaque être rencontré, chaque groupe fréquenté ne reposait entièrement le problème de son identité. Et ce n'est que dans l'insécurité de sa dernière rencontre avec Claudia qu'une relation hors de la détermination collective, pourra mener à un certain bonheur, peut-être.

Mais que vient-il faire au Canada? C'est d'ailleurs la question que lui posent journalistes et interviewers de la radio et de la télévision. Meïr ne donne jamais une réponse complète, se contentant de boutades. Mais il est là 
et veut rester malgré toutes les incertitudes. Certes, désormais tout est possible, il est seul et libre mais il ne parvient pas à calmer tout à fait sa frayeur. Ce désarroi et cette solitude n'arrivent cependant pas à ébranler sérieusement sa détermination, sa volonté de réussir par lui-même. Désormais rien n'est facile car tout est différent. L'Europe différait moins de son Orient natal car il y était mieux préparé: Ici j'étais loin, et je ne parvenais même pas à mesurer la distance, ne sachant où fixer le point de départ. (p. 15). C'est à l'aide du mythe qu'il affronte le réel. Tous les clichés et stéréotypes sur le Canada viennent opportunément calmer ses angoisses. Alors qu'il est transi de froid, il se répète machinalement les topoï positifs de l'hiver québécois du genre le froid vivifie, donne de l'énergie, Canada pays du 20 e siècle, lieu, où tout est possible, terre d'accueil et de liberté. Voilà ce qu'on lui sert tous les jours et qu'il se sert à lui-même alors qu'il cherche désespérément un premier emploi. Mais de vision consciente de son destin Meïr n'en manifeste pas, si ce n'est dans la spontanéité du désespoir ou du bonheur. Dès qu'il gagne quelques dollars en vendant des tissus, il oublie et les doutes refoulés remontent à la surface: J'étais enfin réhabilité. J'allais faire de l'argent. Riche, on me respecterait. C'était l'Amérique. Mais il a beau se les répéter, ces slogans sonnent creux: Il fallait les crier les proclamer à haute voix pour qu'ils fussent convaincants. Il n'est donc pas dupe. Malgré le froid, la solitude, la laideur ambiante, grâce à la chaleur des premières rencontres, les premières amours, il se conforte dans sa détermination même s'il en ignore les raisons. Je ne sais pas pourquoi je suis venu ici, admet-il à la toute fin. Mais cette admission comporte pour la première fois une ébauche de motivation: Nous étions deux naufragés. Nous allions nous noyer ou nous sauver ensemble.

Car cette recherche du pays est, comme dans les Fruits arrachés, tout au long médiatisée par des figures féminines, chacune des «conquêtes" présentant une facette de la Fiancée promise. Magda, la serveuse hongroise qui habite la même pension, lui apporte la première chaleur humaine et brise pour quelques instants sa solitude. Rose, la petite Irakienne, il ne pourra la toucher, lui faire l'amour, car, à cette petite fille de Bagdad, se superposent les images de sa sœur, des cousines, des sœurs d'amis, la conception arabe de la femme. Chacune représente aussi une facette de la ville. Evelyne, Juive polonaise, l'initie au milieu cultivé de McGill alors qu'Helen représente la solidité et l'assurance des Canadiens anglais. Avec Diane, la Canadienne française de Trois-Rivières, les choses vont beaucoup plus loin mais la chaleur qui l'a réconforté n'arrive pas à faire disparaître doutes et angoisses:

J'avais peur de m'interroger sur la nature de nos rapports, nos sentiments. Nous étions bien ensemble et obtenions de nos corps les réponses immédiates que nous prolongions dans une intimité muette comme pour taire toute parole. Nous expliquer? Ce serait la fin, ou le malentendu accepté recherché comme un refuge.

C'est Diane qui le quitte pour étudier à Paris. La dernière femme du roman n'est pas elle non plus la fiancée promise, elle est la promesse. Il a connu plusieurs femmes comme si c'était une course: Je parcourais une étape et hop! une autre. Ce n'est jamais terminé. Mais Claudia, elle aussi venue 
d'ailleurs, est en marche comme lui, elle ira où il ira. Tels deux naufragés ils allaient se noyer ou se sauver ensemble. Tous deux conscients du vide, ils n'en ont pas peur. La première, elle ne tente pas de le définir, de l'identifier à un groupe;

Un piètre explorateur. Je suis venu ici pour faire ma vie comme on dit et j'ai vite découvert que les images que l'on projette de moi-même bouleversent l'idée que je me faisais de la vie. On cherche constamment à me chasser, à me mettre dans des cadres. Et quand je me sens prisonnier, pris au piège, j'abandonne. Je laisse tomber. Je me tais.

À Montréal comme à Paris, on cherche tout au long à le définir, à le situer dans un ensemble. Minoritaire chez les minoritaires, il ne veut renoncer à rien mais n'accepte pas de représenter une réalité même si pour échapper au risque de dissolution, il fait semblant, comme un mannequin dans une vitrine.

Dès les premières lignes, ce Juif polonais qui fait route avec lui dans le train l'embarrasse en se disant heureux de partager avec lui une communauté de destin. Il refuse dans les premières semaines d'utiliser les services du bureau de placement de la communauté juive, préférant faire seul toutes les démarches. Pour les Canadiens français, il est un phénomène,puisque Juif et francophone, réalités difficilement conciliables. Aux préjugés classiques des gens ordinaires (commerçant riche, propriétaire de l'immeuble où il est "chambreur» à $8 \$$ par semaine!) à la vision théologique des prêtres s'ajoute, chez les intellectuels québécois, une volonté d'en faire un allié naturel contre les anglophones ou plus simplement le témoin de leur libéralisme. Flatté d'être invité à la réunion de fondation d'un groupe vișant à la diffusion de la langue et la culture française, Meïr est un peu désespéré du climat théologique ambiant même si certains échappent à cet envahissement du discours religieux. Mais par-dessus tout, il ne représente personne, ne veut pas parler au nom d'un peuple qu'il ne connaît pas. Je ne veux pas qu'on me force à être juif, à être un certain genre deJuif.

Mais il n'y échappe pas. Non seulement doit-il recourir à la communauté pour dénicher un emploi mais il travaillera pour la communauté. Et pourtant pour celle-ci aussi il est un corps étranger, tant par ses origines que par ses choix. Car la communauté juive de Montréal en 1954 est presque totalement ashkenase d'Europe de l'Est et presque tous parlent yiddish et anglais; lui, sépharade et arabe, ne parle pas yiddish et a choisi la culture française. Dans cette ville divisée dont les parties sont si étrangères l'une à l'autre, il se demande qui choisir. À un récital du jeune Léonard Cohen, il s'indigne que l'on n'ait même pas pensé à inviter des journalistes et des écrivains canadiens-français. Les Anglophones non juifs le repoussent quant à eux spontanément vers la communaué juive hors de laquelle il n'a pas d'existence. Les seuls auxquels il ne soit pas étranger sont les quelques Juifs irakiens échoués à Montréal. Quel que soit le plaisir de retrouver la langue, la nourriture, les rites, il se rend bien compte qu'il a bien peu à partager avec ces commerçants et artisans qui peuvent difficilement comprendre ses préoccupations intellectuelles et littéraires. 
Le héros des Fruits arrachés et de la Fiancée promise n'est pas uniquement le Meïr de 1948 ou de 1954. C'est aussi, et peut-être surtout, l'écrivain de 1977 et de 1983. Dans cette reconstitution du passé, il y a l'expérience des illusions perdues et des promesses partiellement réalisées. Si l'auteur d'Adieu Babylone et du Réel et le théâtral pouvait encore croire en une vision globalisante du monde, mettre son espoir dans une réalisation de la promesse, celui des derniers romans et essais a vu l'éclatement de certaines illusions. L'Amérique, et toute la littérature actuelle en témoigne, n'est pas le lieu où coule le lait et le miel. Kattan critique littéraire a traqué ce qui reste de la promesse faite à l'immigrant: il y a trouvé chez les écrivains américains, particulièrement les juifs et les noirs, une évasion dans les drogues et un refermement sur le ghetto. L'auteur de le Désir et le pouvoir ne voit dans la modernité occidentale que les signes d'altérations du désir. Mais il a aussi appris la patience, compris que l'attente n'était pas l'exil. La Bible lui a appris que l'Occident et particulièrement l'Amérique ne sont pas, malgré l'attrait irrésistible qu'ils exercent, la réalisation de la promesse faite à l'Orient mais un état de cette promesse.

Tout dès lors devient choix, découverte, acquisition; appartenant à une minorité il se reconnaît désormais minoritaire à ses propres conditions: C'est par respect pour le monde où je faisais mon entrée que je voulais sauvegarder de l'ancien le plus précieux, celui qui plonge dans l'intimité. C'est dans la tension qu'il aménage sa place dans cette langue nouvelle, acceptant l'imbrication des langues et des cultures et refusant leur cohabitation.

Mais le refus qu'oppose Meïr à être défini par les autres pour son appartenance à un groupe n'est pas dénué de toute ambiguïté. Si Meïr est malheureux de faire de ses origines et de ses convictions une profession, une carrière, Naïm le romancier, en fait la matière de son récit. Le long cheminement vers le pays de la promesse n'est-ce-pas d'abord le dur itinéraire de l'écrivain qui par son désir construit l'œuvre, seule capable de le faire exister dans la complicité de l'échange avec l'autre. En allant jusqu'au plus douloureux de sa spécificité, Kattan atteint à l'universel. Il apporte à la littérature d'ici une contribution essentielle, anamorphose nous renvoyant une vision exacerbée de cette identité tant recherchée mais jamais atteinte.

Paris, juillet 1985

1. Sauf le $1^{\text {er }}$ chapitre de l'édition canadienne. Cette coquille disparait dans l'édition Julliard.

2. Les chapitres se terminent par un __ et les sous-chapitres sont divisés par des $* * *$.

3. S'il fallait s'en tenir à une vision purement autobiographique, il faudrait peut-être expliquer comment, entre autres, le récit des Fruits arrachés se déroule sur une période de deux ans (circ. 1947 à 1949) alors que l'arrivée en Amérique de l'auteur ne se produit qu'en 1954. Le séjour en Europe de sept ans a été ici réduit à deux ans. 